\title{
Entanglement entropy scaling in solid-state spin arrays via capacitance measurements
}

\author{
Leonardo Banchi, Abolfazl Bayat, and Sougato Bose \\ Department of Physics and Astronomy, University College London, Gower Street, WC1E 6BT London, United Kingdom
}

(Received 22 August 2016; revised manuscript received 2 November 2016; published 27 December 2016)

\begin{abstract}
Solid-state spin arrays are being engineered in varied systems, including gated coupled quantum dots and interacting dopants in semiconductor structures. Beyond quantum computation, these arrays are useful integrated analog simulators for many-body models. As entanglement between individual spins is extremely short ranged in these models, one has to measure the entanglement entropy of a block in order to truly verify their many-body entangled nature. Remarkably, the characteristic scaling of entanglement entropy, predicted by conformal field theory, has yet to be measured. Here, we show that with as few as two replicas of a spin array, and capacitive double-dot singlet-triplet measurements on neighboring spin pairs, the above scaling of the entanglement entropy can be verified. This opens up the controlled simulation of quantum field theories, as we exemplify with uniform chains and Kondo-type impurity models, in engineered solid-state systems. Our procedure remains effective even in the presence of typical imperfections of realistic quantum devices and can be used for thermometry, and to bound entanglement and discord in mixed many-body states.
\end{abstract}

DOI: 10.1103/PhysRevB.94.241117

Introduction. More than two decades of active research in quantum information processing has promoted various quantum technologies, which are believed to have resulted in a new industrial revolution [1]. One of the major goals, which dates back to Feynman [2], is to simulate complex interacting quantum systems, which are intractable with classical computers, with an engineered and controllable quantum device, the so-called quantum simulator [3]. Unlike general-purpose quantum computers, which are supposed to be programmable to achieve different tasks, quantum simulators are designed for a specific goal, which make them easier to realize. Indeed, so far cold atoms [4] and ions [5] have been used for successfully simulating certain tasks. Nevertheless, solid-state-based quantum simulators are still highly in demand due to the fact that (i) they provide more versatile types of interactions and stronger couplings compared to cold atoms and ions, and (ii) the quest towards miniaturization in electronics has reached the quantum level, making solid-state quantum devices feasible [6].

Much theoretical research has been conducted to understand the highly entangled structures appearing in the ground state of quantum many-body systems [7]. For a given bipartition $A$ and $B$ of the whole system, which is assumed to be in the pure state $\rho_{A B}=\left|\psi_{A B}\right\rangle\left\langle\psi_{A B}\right|$, the entanglement entropy is quantified by $S_{\alpha}\left(\rho_{A}\right)=S_{\alpha}\left(\rho_{B}\right)$, where $\rho_{A}=\operatorname{Tr}_{B} \rho_{A B}$ and $S_{\alpha}$ is the Renyi entropy, defined as

$$
S_{\alpha}(\rho)=\frac{1}{1-\alpha} \log \operatorname{Tr}\left[\rho^{\alpha}\right],
$$

for different values of $\alpha$. When $\alpha \rightarrow 1$, the Renyi entropy reduces to the von Neumann entropy $S_{1}(\rho)=-\operatorname{Tr}[\rho \log \rho]$. The importance of the entanglement entropy is twofold: (i) It quantifies the entanglement between $A$ and $B$; and (ii) the discovery of its area law dependence in noncritical systems has immensely contributed to the development of efficient approximation techniques [8] for describing manybody systems. On the other hand, in critical one-dimensional systems with open boundary conditions, conformal field theory analysis shows that there is a logarithmic correction, as

$$
S_{\alpha}(x)=\frac{c}{12}\left(1+\frac{1}{\alpha}\right) \log \left[\frac{2 N}{\pi} \sin \left(\frac{\pi x}{N}\right)\right]+\kappa_{\alpha},
$$

where $x$ is the size of the contiguous block $A$ starting at one end of system, and $N$ is the total size. When $N \gg x \gg 1$, the usual scaling $S_{\alpha} \propto \log x$ is obtained. This formula is very general and the central chargec only depends on the universality class of the model, while the constants $\kappa_{\alpha}$ are model dependent [9-11]. In spite of the extensive theoretical literature on entanglement entropy, its experimental measurement is a big challenge. For itinerant bosonic particles it has been proposed $[12,13]$, and recently realized [14], to use beam-splitter operations or a discrete Fourier transform to measure $S_{\alpha}$. Alternatively measuring entropy through quantum shot noise has been proposed $[15,16]$, but has yet to be realized. On the other hand, in nonitinerant spin systems, the situation becomes even more difficult and the only proposal so far is to use spin-dependent switches [17], which are difficult to build.

Here, we put forward a proposal for measuring $S_{\alpha}$ in a spin system without demanding time-dependent particle delocalization or spin-dependent switches. While our setup can be realized in different physical systems, we target it to solid-state systems, such as gated quantum dot chains [18-24] or dopant arrays [6,25-27]. Our procedure is based on well-established singlet-triplet measurements, which are now routinely performed either via charge detection [28] or capacitive radio-frequency reflectometry [29-32].

Measuring entanglement entropy. Our goal is to measure $S_{\alpha}$ for arbitrarily integer values of $\alpha \geqslant 2$. For simplicity, we explain the procedure for $\alpha=2$ and then generalize it for higher values. Inspired by previous alternative proposals $[12,13,17,33,34]$, we make use of two copies of a spin array in the state $\rho_{1} \otimes \rho_{2}$ (ideally, for perfect copies, $\rho_{1}=\rho_{2}$ ). Each copy is identically divided into two complementary blocks: $A_{1}$ and $B_{1}$ for the first copy, $A_{2}$ and $B_{2}$ for the second one (see Fig. 1). Let $x$ be the number of spins in $A_{1}$ (and $A_{2}$ ). We define 


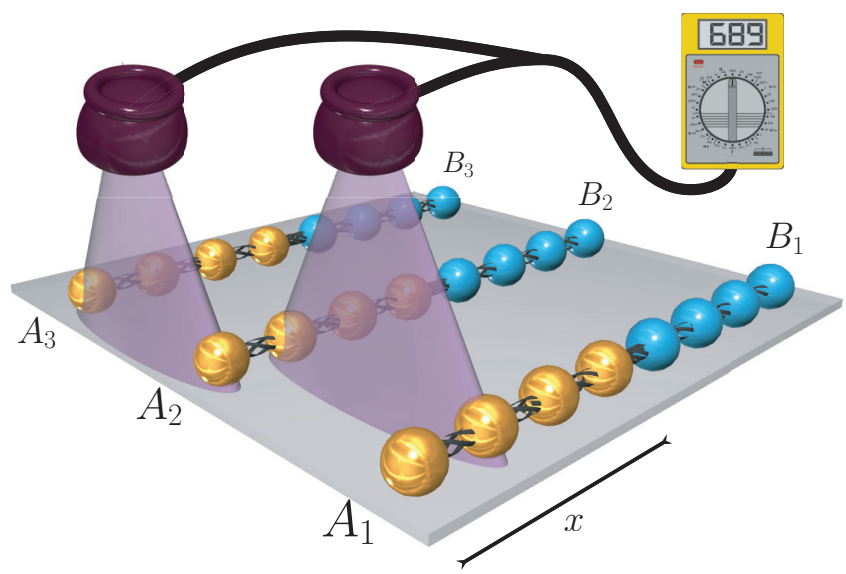

FIG. 1. Scheme for the measurement of the entanglement entropy for $\alpha=3$ using three copies of the same spin chain. Each spin chain is divided into $A_{i}$ (yellow spins) and $B_{i}$ (blue spins). By performing sequential singlet-triplet measurements of a pair of spins in neighboring chains it is possible to estimate $S_{\alpha=3}\left(\rho_{A}\right)$ (see the discussion in the text), which measures the entanglement between $A$ and $B$.

the multispin SWAP operator acting on $A_{1}$ and $A_{2}$ as

$$
P_{12}^{A} \equiv \bigotimes_{\ell=1}^{x} \operatorname{swAP}\left(\ell_{A_{1}}, \ell_{A_{2}}\right)
$$

where $\operatorname{SwAP}\left(\ell_{A_{1}}, \ell_{A_{2}}\right)$ swaps the two spins at the $\ell$ th sites in $A_{1}$ and $A_{2}$. Since all the operators $\operatorname{SWAP}\left(\ell_{A_{1}}, \ell_{A_{2}}\right)$ are commuting, it is simple to show that

$$
\left\langle P_{12}^{A}\right\rangle=\operatorname{Tr}\left[P_{12}^{A} \rho_{1} \otimes \rho_{2}\right]=\operatorname{Tr}\left[\rho_{A_{1}} \rho_{A_{2}}\right]=\operatorname{Tr}\left[\rho_{A}^{2}\right],
$$

where the last equality holds if the two copies are identical, namely, $\rho_{A_{1}} \equiv \rho_{A_{2}} \equiv \rho_{A}$. Therefore, Eq. (4) implies that $S_{2}=$ $-\log \left\langle P_{12}^{A}\right\rangle$ can be obtained via a sequential measurement of pairwise SWAP operators acting on the different spins of $A_{1}$ and $A_{2}$, as shown in Fig. 1.

The above procedure can be generalized to higher integer values of $\alpha$ by considering $\alpha$ copies of the spin array in the state $\rho^{\otimes \alpha}=\bigotimes_{\ell=1}^{\alpha} \rho_{\ell}$ (where ideally all the $\rho_{\ell}$ 's are equal). Remarkably, sequential measurements of multispin SWAP operators acting on neighboring copies $a$ and $a+1$, namely, $P_{a, a+1}^{A}$, are sufficient to measure the Renyi entropy. This is simple, but not trivial, as better explained in the Supplemental Material [35], because some $P_{(a, a+1)}^{A}$ 's for different $a$ are noncommuting. However, we show that the simple sequential measurement, exemplified also in Fig. 1, corresponds to the measurement of the operator $P_{12 \ldots \alpha}^{(A)}$, which is defined recursively by the formula

$$
P_{12 \ldots \alpha}^{A}=\frac{P_{\alpha, \alpha-1}^{A} P_{1 \ldots \alpha-1}^{A}+P_{1 \ldots \alpha-1}^{A} P_{\alpha, \alpha-1}^{A}}{2} .
$$

For example, for $\alpha=3$ this reduces to $P_{123}^{A}=\left(P_{23}^{A} P_{12}^{A}+\right.$ $\left.P_{12}^{A} P_{23}^{A}\right) / 2$ and $\left\langle P_{123}^{A}\right\rangle=\left(\operatorname{Tr}\left[\rho_{A_{1}} \rho_{A_{2}} \rho_{A_{3}}\right]+\operatorname{Tr}\left[\rho_{A_{1}} \rho_{A_{3}} \rho_{A_{2}}\right]\right) / 2$, so that for perfect copies $\left\langle P_{123}^{A}\right\rangle=\operatorname{Tr}\left[\rho_{A}^{3}\right]$. In general, using Eq. (1), we have $S_{\alpha}\left(\rho_{A}\right)=(1-\alpha)^{-1} \log \left\langle P_{12 \ldots \alpha}^{A}\right\rangle$. We stress that $P_{12 \ldots \alpha}^{A}$ is ultimately written in terms of nearest-neighbor multispin SWAP operators $P_{(a, a+1)}^{A}$. This makes the procedure scalable in the laboratory as one has to first measure $P_{12}^{A}$, then $P_{23}^{A}$, and so forth until $P_{\alpha-1, \alpha}^{A}$.

Solid-state spin chains. When exactly one electron is trapped in each quantum dot, the interactions between confined electrons in quantum dot arrays are restricted to the spin sector, and are described by the Heisenberg Hamiltonian

$$
H=\sum_{k=1}^{N-1} J_{k} \boldsymbol{\sigma}_{k} \cdot \boldsymbol{\sigma}_{k+1},
$$

where $J_{k}$ is the exchange coupling between neighboring sites and $\sigma_{k}=\left(\sigma_{k}^{x}, \sigma_{k}^{y}, \sigma_{k}^{z}\right)$ is the vector of Pauli operators acting on site $k$. The couplings $J_{k}$ can be locally tuned by appropriately changing the local gate voltages. The system can be initialized into its ground state either by cooling, when temperatures is below its energy gap, or using an adiabatic-type evolution [36], when the temperature is higher.

Singlet-triplet measurements on two electrons trapped in adjacent quantum dots is now a well-established technique for spin measurements in solid-state physics [28,30-32,37]. In quantum mechanical language the singlet-triplet measurements on a pair of electrons in dots $a$ and $b$ correspond to projective measurements of the SWAP operator, as one can show

$$
\operatorname{SWAP}(a, b)=\sum_{\mu= \pm, 0}\left|t_{\mu}\right\rangle\left\langle t_{\mu}|-| s\right\rangle\langle s|=\frac{\mathbb{1}+\boldsymbol{\sigma}_{a} \cdot \boldsymbol{\sigma}_{b}}{2},
$$

where $|s\rangle=\left(\left|\uparrow_{a} \downarrow_{b}\right\rangle-\left|\downarrow_{a} \uparrow_{b}\right\rangle\right) / \sqrt{2}$ is the singlet state, and $\left|t_{+}\right\rangle=\left|\uparrow_{a} \uparrow_{b}\right\rangle,\left|t_{0}\right\rangle=\left(\left|\uparrow_{a} \downarrow_{b}\right\rangle+\left|\downarrow_{a} \uparrow_{b}\right\rangle\right) / \sqrt{2}$, and $\left|t_{-}\right\rangle=$ $\left|\downarrow_{a} \downarrow_{b}\right\rangle$ are the triplet states. The outcome of this measurement is either +1 for triplet outcomes, and -1 for the singlet one.

By comparing Eqs. (7) and (3) it is now clear that, for any given bipartition, we can use a sequence of singlet-triplet measurements to obtain the outcome of the operators $P_{1 \ldots \alpha}^{A}$ and thus compute all the Renyi entropies $S_{\alpha}$ for all integer $\alpha \geqslant 2$. As described before, and shown also in Fig. 1, the total number of singlet-triplet measurements to be performed for a single outcome is $x \alpha$, where $x$ is the number of spins in subsystem $A$. To measure $P_{1 \ldots \alpha}^{A}$ we first switch off the $J_{k}$ 's within each array, and then lower the barriers between pairs of spins in two different arrays to perform the singlet-triplet measurements. A recently developed multiplexer structure [38] containing two parallel arrays of quantum dots is a promising setup, which can be adapted for measuring $S_{2}$ with our proposed mechanism. Motivated by this operating device, and for the sake of simplicity, in the rest of this Rapid Communication we focus on $\alpha=2$. Numerical results are obtained with either density matrix renormalization group (DMRG) or exact diagonalization for short chains.

Application 1: Conformal field theory in the laboratory. We first present how field theory predictions, given in Eq. (2), can be verified for a uniform chain where $J_{k}=J$, for all $k$ 's. In the thermodynamic limit $N \rightarrow \infty$ it is known that the central charge is $c=1$. In Fig. 2(a) we plot the Renyi entropy $S_{2}$ as a function of $\log \left[\frac{2 N}{\pi} \sin \left(\frac{\pi x}{N}\right)\right]$ in a chain of length $N=60$. For open boundary conditions, finite-size effects are known [9] to give rise to an alternating behavior of $S_{2}(x)=S_{2}^{(U)}+$ $(-1)^{x} S_{2}^{(A)}$. Using the methodology of Ref. [39] we extract the uniform part $S_{2}^{(U)}$, which is dominant for $N \rightarrow \infty$ and 

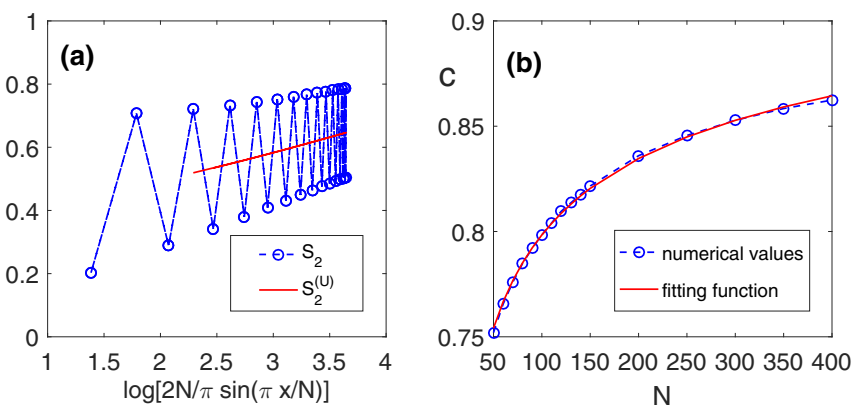

FIG. 2. (a) Scaling of $S_{2}$ and its uniform part $S_{2}^{(U)}$ in terms of different sizes of block $A$, for a chain of $N=60$. (b) Scaling of the central charge $c$ as a function of length $N$.

follows the scaling of (2). In Fig. 2(a) we also plot $S_{2}^{(U)}$ in red colors, showing perfect linear scaling. From the slope of this line we can extract the central charge $c$, which asymptotically approaches its thermodynamic limit value, $c=1$. This can be seen in Fig. 2(b), where we also plot the fitting function $c=$ $1-0.7536 N^{-0.2848}$. Such slow convergence is due to finitesize corrections to the field theory predictions [40], which here, for simplicity, we have absorbed into the definition of $c$.

Application 2: Impurity entanglement entropy. Introducing one or more impurities in the system can change its behavior dramatically. A paradigmatic example is the single-impurity Kondo model [41] in which a single impurity in a gapless system creates a length scale $\xi$, known as the Kondo length. The scaling features of Kondo physics can be captured by a spin chain emulation of this model [39]. This is described by Eq. (6), where $J_{1}=J^{\prime}$, while all other couplings remain uniform $J_{k}=J$ (for $k \geqslant 2$ ). Moreover, the length scale is determined by $J^{\prime}$ as $\xi \propto e^{g / J^{\prime}}$ for some constant $g$. The presence of the impurity modifies the scaling of Eq. (2) when $x<\xi$. In order to capture the impurity contribution of the entanglement entropy we extend the ansatz of Ref. [39] for $S_{1}$ to generic $S_{\alpha}$ and define the impurity entanglement entropy as

$$
S_{\alpha}^{(\mathrm{imp})}(x, N, \xi)=S_{\alpha}^{(U)}(x, N, \xi)-S_{\alpha}^{(U)}(x-1, N-1),
$$

where $S_{\alpha}^{(U)}(x, N, \xi)$ is the Renyi entropy of a block of size $x$ in a chain of length $N$ and impurity coupling $J^{\prime}$, which determines $\xi$, while $S_{\alpha}^{(U)}(x-1, N-1)$ represents the bulk contribution of the uniform chain when the impurity is removed. In Fig. 3(a) we plot the $S_{2}(x, N, \xi)$ and its uniform part $S_{2}^{(U)}$ in a chain of length $N=60$. The bulk contribution of the uniform chain, i.e., $S_{2}(x-1, N-1)$, and its uniform part $S_{2}^{(U)}(x-1, N-1)$ are plotted in Fig. 3(b). The qualitative difference between Figs. 3(a) and 3(b) is due to the different parities (i.e., even and odd) of the chains.

The emergence of the length scale implies that $S_{2}^{(\text {imp) }}(x, N, \xi)$ is only a function of the ratios $S_{2}^{(\text {imp })}(x / N, N / \xi)$. To verify this scaling we fix $N / \xi$ and plot $S_{2}^{(\text {imp) }}$ as a function of $x / N$ for different lengths $N$. To keep $N / \xi$ fixed, $J^{\prime}$ has to be tuned according to Ref. [42]. The results are shown in Fig. 3(c) where, as predicted, the curves of different chains collapse onto each other. Although the data collapse becomes better by increasing the system size, Fig. 3(c) shows that the scaling predictions can be captured even in relatively small chains.

Application 3: Entanglement spectrum. For any pure state $\rho_{A B}$ the eigenvalues of $\rho_{A}$ are called the entanglement spectrum [43], whose analysis is important to characterize quantum phase transitions $[44,45]$. The eigenvalues of $\rho_{A}$ are the roots of $q(\lambda)=\operatorname{det}\left(\lambda \mathbb{1}-\rho_{A}\right)$, which can be written as $q(\lambda)=\sum_{k} g_{k} \lambda^{k}$. According to Ref. [46], the coefficients $g_{k}$ can be obtained algorithmically from $\operatorname{Tr}\left[\rho_{A}^{\alpha}\right]$ for $\alpha=1, \ldots, k$. Since these traces can be measured with our procedure, one can build $q(\lambda)$ and hence obtain the full entanglement spectrum. Clearly, given a maximum number of copies $\alpha_{\max }$, one can find the entanglement spectrum for block sizes as large as $x=\log _{2} \alpha_{\max }$.

Application 4: Thermometry via purity measurement. One of the biggest challenges in solid-state experiments is to measure the true temperature of electrons, as it is normally higher than the temperature of the refrigerator. Remarkably, our scheme enables us also to measure the electronic temperature via singlet-triplet measurements, assuming that the system is in a thermal state $\rho_{\beta}=e^{-\beta H} / Z$. Our approach is based on three distinctive features of engineered solid-state structures: (i) The exchange integral $J$ can be varied; (ii) the purity $\mathcal{P}(\beta, J)=\operatorname{Tr}\left[\rho_{\beta}^{2}\right]=e^{-S_{2}\left(\rho_{\beta}\right)}$ can be measured with our scheme by taking two copies and $x=N$; and (iii) computing
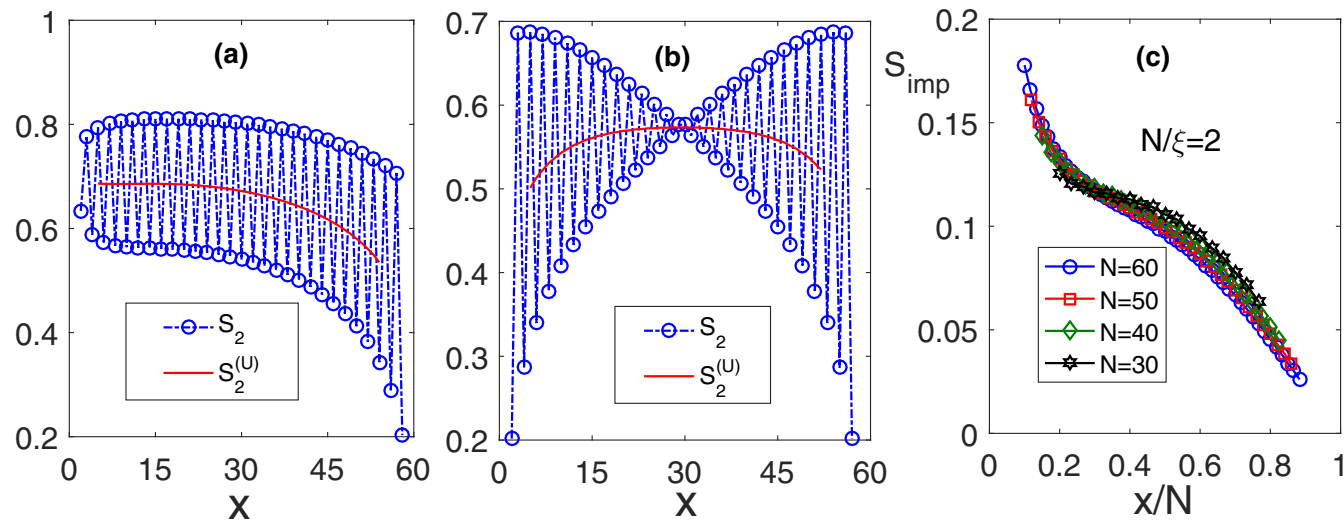

FIG. 3. (a) The Renyi entropy $S_{2}(x, N, \xi)$ and its uniform part $S_{2}^{(U)}$ in a chain of length $N=60$. (b) The bulk Renyi entropy $S_{2}(x-1, N-1)$ and its uniform part $S_{2}^{(U)}(x-1, N-1)$ in a homogeneous chain of 59 sites without impurity. (c) Data collapse for different chains when $J^{\prime}$ is tuned to keep $N / \xi=2$. 

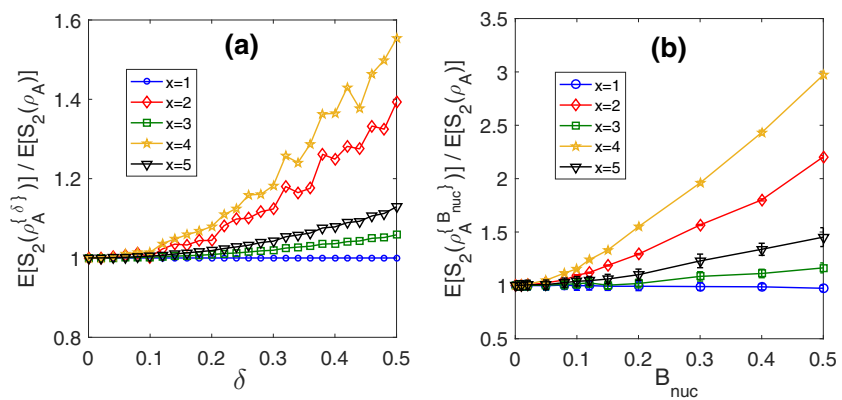

FIG. 4. (a) Average $\mathbb{E}\left[S_{2}\left(\rho_{A}^{\{\delta\}}\right)\right]$ over 1000 random sets of couplings $J_{k}^{(j)}=J_{k}\left(1+\epsilon_{k}^{(j)}\right)$, where $j=1,2$ refers to the different copies, $J_{k}=J$, and $\epsilon_{k}^{(j)}$ is uniformly distributed in $[-\delta,+\delta]$ for different block sizes $x$. (b) Monte Carlo simulation of $\mathbb{E}\left[S_{2}\left(\rho_{A}^{\left\{B_{\text {nuc }}\right\}}\right)\right]$ for the effect of random fields $B_{k}^{(j)}$ as a function of the strength of the hyperfine interaction $B_{\text {nuc }}$. In both figures the entropies are normalized with respect to the error-free case $S_{2}\left(\rho_{A}\right)$, and $N=10$.

the energy expectation $E(\beta, J)=\operatorname{Tr}\left[H \rho_{\beta}\right]$ is reduced to singlet-triplet measurements on neighboring sites of one of the arrays, owing to Eqs. (7) and (6). A simple calculation reveals that $\frac{\partial \mathcal{P}(\beta, J)}{\partial J}=\frac{\beta}{J}[2 E(\beta, J)-E(\beta, 2 J)] \mathcal{P}(\beta, J)$. Aside from $\beta$, all the quantities in the above equality can be measured either directly [namely, $\mathcal{P}(\beta, J)$ and $E(\beta, J)$ ] or through the variation of $J$ [namely, $\frac{\partial \mathcal{P}(\beta, J)}{\partial J}$ and $\left.E(\beta, 2 J)\right]$. In summary, owing to the above equality, using different singlet-triplet measurements with different values of $J$, it is possible to infer $\beta$ and thus the temperature.

Application 5: Bounding entanglement and discord in mixed states. The Renyi entropy of a block $A$ is a measure of entanglement between $A$ and $B$ only if $\rho_{A B}$ is a pure state. However, we show that it is still possible to bound the amount of entanglement and discord also for mixed states by measuring both $S_{\alpha}\left(\rho_{A}\right)$ and $S_{\alpha}\left(\rho_{A B}\right)$. The distillable entanglement $E_{D}$, an operational entanglement measure, satisfies the hashing inequality [47], $E_{D} \geqslant \max _{X=A, B} I_{1}(X)$, where $I_{1}(X)=S_{1}\left(\rho_{X}\right)-S_{1}\left(\rho_{A B}\right)$. Similarly, for the quantum discord $D(A \mid B)$, which is an asymmetric measure of quantum correlations between $A$ and $B$ [48], it is known that $D(A \mid B) \geqslant I_{1}(A)$ and similarly $D(B \mid A) \geqslant I_{1}(B)$ [49]. The von Neumann entropy $S_{1}$ can be extrapolated [50] from $S_{\alpha}$ for different integers $\alpha \geqslant 2$, which can be measured with our scheme. However, we show that $I_{1}$ can also be bounded by directly measuring $S_{2}\left(\rho_{A}\right)$ and $S_{2}\left(\rho_{A B}\right)$, which require only two replicas. Indeed, since $S_{2}(\rho) \leqslant S_{1}(\rho) \leqslant f\left[S_{2}(\rho)\right]$, where $f$ is given in Ref. [51], we obtain $I_{1}(X) \geqslant I_{2}(X)$, where $I_{2}(X)=S_{2}\left(\rho_{X}\right)-f\left[S_{2}\left(\rho_{A B}\right)\right]$. For either $X=A, B, I_{2}(X)$ thus provides a measurable lower bound to entanglement and discord.

Imperfections. Realistic experimental imperfections may introduce errors, e.g., by making the different copies nonidentical. Our protocol provides $\operatorname{Tr}\left[\rho_{A_{1}} \rho_{A_{2}}\right]$, as given in
Eq. (4), which may deviate from the ideal case $\operatorname{Tr}\left[\rho_{A}^{2}\right]$. Indeed, imperfect fabrication may result in random couplings $J_{k}^{(j)} \rightarrow J_{k}\left(1+\epsilon_{k}^{(j)}\right)$, where $j$ is the index of different copies and $\epsilon_{k}^{(j)}$ is a random number uniformly distributed between $[-\delta,+\delta]$. In Fig. 4(a) we calculate the average $\mathbb{E}\left[S_{2}\left(\rho_{A}^{\{\delta\}}\right)\right]$ over 1000 random sets of couplings for different block sizes $x$, normalized with respect to its value at $\delta=0$. As the figure shows, the average entropy increases by increasing $\delta$. Moreover, up to $\delta=10 \%$, the outcomes are almost indistinguishable from the error-free case.

The second source of imperfections is due to the hyperfine interaction with the nuclear spins in the bulk, which effectively introduces an extra term $\sum_{k} \boldsymbol{B}_{k}^{(j)} \cdot \boldsymbol{\sigma}_{k}$ in the Hamiltonian (6), where each component of the random fields has a normal distribution with zero mean and variance $B_{\text {nuc }}$. Unlike the randomness in the couplings, which is constant over different experiments being due to the fabrication, the effective random fields are different in any experimental repetition. To realistically model this, we perform a Monte Carlo simulation of real experimental outcomes (see the Supplemental Material for details). The results are shown in Fig. 4(b) for different block sizes $x$. For realistic values of $B_{\text {nuc }}=0.1 J$ [28], we see that the entanglement entropy is only slightly affected by hyperfine interactions. The increasing trend of the entropy as a function of the noise, which is consistent with Ref. [52], is further discussed in the Supplemental Material.

Conclusions. We propose a scheme to experimentally measure the entanglement between blocks in engineered solid-state quantum devices. Our procedure is based on singlet-triplet measurements which are routinely performed in quantum dot systems. All the Renyi entanglement entropies $S_{\alpha}$ for integer $\alpha \geqslant 2$ can be measured via $\alpha$ replicas of the system. Although for uniform chains the convergence of the central charge is slow, the logarithmic scaling predictions can already be verified with reasonably small system sizes (§60). Moreover, in the Kondo impurity model we found that the impurity contribution in the Renyi entropy satisfies a universal scaling law. Despite the fact this law has been obtained in the thermodynamic limit, remarkably, it can be observed for chains as small as $N \gtrsim 30$. In addition, our scheme enables the measurement of the purity of the whole system, which allows one to measure the true temperature of electrons in a thermal state. Our procedure remains effective even in the presence of typical imperfections due to imperfect fabrication and hyperfine interactions. Although our scheme has been targeted to quantum dot arrays, the same protocol can also be realized in other systems, such as dopants in silicon [26].

Acknowledgments. L.B. and S.B. have received funding from the European Research Council under the European Union's Seventh Framework Programme (FP/20072013)/ERC Grant Agreement No. 308253. A.B. and S.B. acknowledge EPSRC Grant No. EP/K004077/1.
[1] J. P. Dowling and G. J. Milburn, Philos. Trans. R. Soc., A 361, 1655 (2003).

[2] R. P. Feynman, Int. J. Theor. Phys. 21, 467 (1982).
[3] J. I. Cirac and P. Zoller, Nat. Phys. 8, 264 (2012).

[4] I. Bloch, J. Dalibard, and S. Nascimbene, Nat. Phys. 8, 267 (2012). 
[5] R. Blatt and C. Roos, Nat. Phys. 8, 277 (2012).

[6] J. Salfi, J. Mol, R. Rahman, G. Klimeck, M. Simmons, L. Hollenberg, and S. Rogge, Nat. Commun. 7, 11342 (2016).

[7] L. Amico, R. Fazio, A. Osterloh, and V. Vedral, Rev. Mod. Phys. 80, 517 (2008).

[8] J. Eisert, M. Cramer, and M. B. Plenio, Rev. Mod. Phys. 82, 277 (2010).

[9] P. Calabrese, M. Campostrini, F. Essler, and B. Nienhuis, Phys. Rev. Lett. 104, 095701 (2010).

[10] P. Calabrese and J. Cardy, J. Stat. Mech.: Theory Exp. (2004) P06002.

[11] M. Fagotti and P. Calabrese, J. Stat. Mech.: Theory Exp. (2011) P01017.

[12] C. M. Alves and D. Jaksch, Phys. Rev. Lett. 93, 110501 (2004).

[13] A. J. Daley, H. Pichler, J. Schachenmayer, and P. Zoller, Phys. Rev. Lett. 109, 020505 (2012).

[14] A. M. Kaufman, M. E. Tai, A. Lukin, M. Rispoli, R. Schittko, P. M. Preiss, and M. Greiner, Science 353, 794 (2016).

[15] H. F. Song, S. Rachel, C. Flindt, I. Klich, N. Laflorencie, and K. Le Hur, Phys. Rev. B 85, 035409 (2012).

[16] I. Klich and L. Levitov, Phys. Rev. Lett. 102, 100502 (2009).

[17] D. A. Abanin and E. Demler, Phys. Rev. Lett. 109, 020504 (2012).

[18] D. Loss and D. P. DiVincenzo, Phys. Rev. A 57, 120 (1998).

[19] R. Hanson, L. Kouwenhoven, J. Petta, S. Tarucha, and L. Vandersypen, Rev. Mod. Phys. 79, 1217 (2007).

[20] T. Baart, N. Jovanovic, C. Reichl, W. Wegscheider, and L. Vandersypen, Appl. Phys. Lett. 109, 043101 (2016).

[21] T. Nakajima, M. R. Delbecq, T. Otsuka, S. Amaha, J. Yoneda, A. Noiri, K. Takeda, G. Allison, A. Ludwig, A. D. Wieck et al., arXiv: 1604.02232 .

[22] T. Ito, T. Otsuka, S. Amaha, M. R. Delbecq, T. Nakajima, J. Yoneda, K. Takeda, G. Allison, A. Noiri, K. Kawasaki et al., arXiv: 1604.04426.

[23] D. Zajac, T. Hazard, X. Mi, E. Nielsen, and J. Petta, Phys. Rev. Appl. 6, 054013 (2016).

[24] A. Noiri, J. Yoneda, T. Nakajima, T. Otsuka, M. R. Delbecq, K. Takeda, S. Amaha, G. Allison, A. Ludwig, A. D. Wieck et al., Appl. Phys. Lett. 108, 153101 (2016).

[25] B. E. Kane, Nature (London) 393, 133 (1998).

[26] F. A. Zwanenburg, A. S. Dzurak, A. Morello, M. Y. Simmons, L. C. Hollenberg, G. Klimeck, S. Rogge, S. N. Coppersmith, and M. A. Eriksson, Rev. Mod. Phys. 85, 961 (2013).

[27] M. Fuechsle and M. Y. Simmons, in Single-Atom Nanoelectronics, edited by E. Prati and T. Shinada (Pan Stanford Publishing, Singapore, 2013), p. 61.
[28] J. R. Petta, A. C. Johnson, J. M. Taylor, E. A. Laird, A. Yacoby, M. D. Lukin, C. M. Marcus, M. P. Hanson, and A. C. Gossard, Science 309, 2180 (2005).

[29] M. House, T. Kobayashi, B. Weber, S. Hile, T. Watson, J. van der Heijden, S. Rogge, and M. Simmons, Nat. Commun. 6, 8848 (2015).

[30] K. Petersson, C. Smith, D. Anderson, P. Atkinson, G. Jones, and D. Ritchie, Nano Lett. 10, 2789 (2010).

[31] T. Frey, P. J. Leek, M. Beck, A. Blais, T. Ihn, K. Ensslin, and A. Wallraff, Phys. Rev. Lett. 108, 046807 (2012).

[32] J. I. Colless, A. C. Mahoney, J. M. Hornibrook, A. C. Doherty, H. Lu, A. C. Gossard, and D. J. Reilly, Phys. Rev. Lett. 110, 046805 (2013).

[33] P. Horodecki and A. Ekert, Phys. Rev. Lett. 89, 127902 (2002).

[34] J. Cardy, Phys. Rev. Lett. 106, 150404 (2011).

[35] See Supplemental Material at http://link.aps.org/supplemental/ 10.1103/PhysRevB.94.241117 for more details on the derivation of Eq. (5) and on the error analysis of Fig. 4.

[36] U. Farooq, A. Bayat, S. Mancini, and S. Bose, Phys. Rev. B 91 , 134303 (2015).

[37] M. Delbecq, T. Nakajima, P. Stano, T. Otsuka, S. Amaha, J. Yoneda, K. Takeda, G. Allison, A. Ludwig, A. Wieck et al., Phys. Rev. Lett. 116, 046802 (2016).

[38] R. Puddy, L. Smith, H. Al-Taie, C. Chong, I. Farrer, J. Griffiths, D. Ritchie, M. Kelly, M. Pepper, and C. Smith, Appl. Phys. Lett. 107, 143501 (2015).

[39] E. S. Sørensen, M.-S. Chang, N. Laflorencie, and I. Affleck, J. Stat. Mech.: Theory Exp. (2007) P08003.

[40] J. C. Xavier and F. C. Alcaraz, Phys. Rev. B 85, 024418 (2012).

[41] I. Affleck, Quantum Impurity Problems in Condensed Matter Physics (Oxford University Press, Oxford, U.K., 2008).

[42] A. Bayat, P. Sodano, and S. Bose, Phys. Rev. B 81, 064429 (2010).

[43] H. Li and F. D. M. Haldane, Phys. Rev. Lett. 101, 010504 (2008).

[44] G. De Chiara, L. Lepori, M. Lewenstein, and A. Sanpera, Phys. Rev. Lett. 109, 237208 (2012).

[45] A. Bayat, H. Johannesson, S. Bose, and P. Sodano, Nat. Commun. 5, 3784 (2014).

[46] T. L. Curtright and D. B. Fairlie, arXiv:1212.6972.

[47] I. Devetak and A. Winter, Proc. R. Soc. London, Ser. A 461, 207 (2005).

[48] H. Ollivier and W. H. Zurek, Phys. Rev. Lett. 88, 017901 (2001).

[49] F. F. Fanchini, M. F. Cornelio, M. C. de Oliveira, and A. O. Caldeira, Phys. Rev. A 84, 012313 (2011).

[50] C. De Nobili, A. Coser, and E. Tonni, J. Stat. Mech.: Theory Exp. (2015) P06021.

[51] K. Życzkowski, Open Syst. Inf. Dyn. 10, 297 (2003).

[52] J. C. Getelina, F. C. Alcaraz, and J. A. Hoyos, Phys. Rev. B 93, 045136 (2016) 